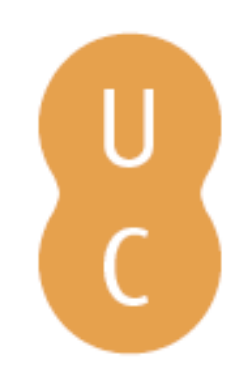

\title{
pompalina
}

\section{Le système mental en tant que composant de l'émotion}

Autor(es): $\quad$ Scherer, Klaus R.; Sangsue, Janique

Publicado por: Imprensa da Universidade de Coimbra; Les Éditions de L'IQRC (Les

URL

persistente: URI:http://hdl.handle.net/10316.2/32655

DOI: $\quad$ DOI:http://dx.doi.org/10.14195/978-989-26-0805-1_1

Accessed : $\quad$ 26-Apr-2023 04:36:44

A navegação consulta e descarregamento dos títulos inseridos nas Bibliotecas Digitais UC Digitalis, UC Pombalina e UC Impactum, pressupõem a aceitação plena e sem reservas dos Termos e Condições de Uso destas Bibliotecas Digitais, disponíveis em https://digitalis.uc.pt/pt-pt/termos.

Conforme exposto nos referidos Termos e Condições de Uso, o descarregamento de títulos de acesso restrito requer uma licença válida de autorização devendo o utilizador aceder ao(s) documento(s) a partir de um endereço de IP da instituição detentora da supramencionada licença.

Ao utilizador é apenas permitido o descarregamento para uso pessoal, pelo que o emprego do(s) título(s) descarregado(s) para outro fim, designadamente comercial, carece de autorização do respetivo autor ou editor da obra.

Na medida em que todas as obras da UC Digitalis se encontram protegidas pelo Código do Direito de Autor e Direitos Conexos e demais legislação aplicável, toda a cópia, parcial ou total, deste documento, nos casos em que é legalmente admitida, deverá conter ou fazer-se acompanhar por este aviso.

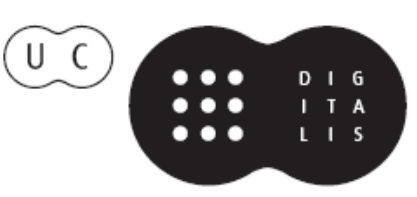


GILLES KIROUAC

Coordonnateur Scientifique

Cognition
et Émotions 
(Página deixada propositadamente em branco) 


\title{
GILLES KIROUAC
}

Coordonnateur Scientifique

\section{Cognition et Émotions}

\author{
AUTEURS \\ Klaus R. Scherer \& Janique Sangsue \\ Pierre Philippot \\ Dario Galati \\ Bernard Rimé \\ Pierre Gosselin \\ Ursula Hess \\ Arvid Kappas \& Jean Descôteaux \\ Pio E. Ricci Bitti \\ Pedro Luzes \\ Lise Fillion \\ Ramon Bayés \\ Coimbra - Imprensa da Universidade


COORDENAÇÃO EDITORIAL

Imprensa da Universidade de Coimbra

CONCEPÇÃO GRÁFICA

António Barros

PAGINAÇÃo

António Resende

[Universidade de Coimbra]

EXECUÇÃO GRÁFICA

G.C. - Gráfica de Coimbra, Lda.

Palheira - Assafarge - Apart. 3068

300I-453 Coimbra Codex

ISBN 972-8704-19-4

ISBN 2-7637-81।8-7

DEPÓSITO LEGAL 210430/04

(C) MAIO 2004, Imprensa DA UnIVERSIDAdE DE COIMBRA

\author{
OBRA PUBLICADA COM O PATROCINIO DO \\ INSTITUTO DE PSICOLOGIA COGNITIVA \\ FCT: FUNDAÇÃO PARA A CIÊNCIA E A TECNOLOGIA \\ MINISTÉRIO DA CIÊNCIA E DO ENSINO SUPERIOR \\ apolo do Programa Operacional CiênCIa, TeCnologia, Inovação \\ DO QUADRO COMUNITÁRIO DE APOIO
}


Klaus R. Scherer \& Janique Sangsue

Université de Genève, Suisse

\section{LE SYSTĖME MENTAL EN TANT QUE COMPOSANT DE L'ÉMOTION}

Le domaine de l'émotion reste encore actuellement l'enfant pauvre des ouvrages de psychologie en langue française. La dernière contribution de taille fut celle de Fraisse (1969) dans son Traité de Psychologie Expérimentale, contribution qui cependant ne constituait qu'un résumé encyclopédique des diverses définitions de l'émotion. Ceci parait surprenant puisque la tradition francophone, avec Dumas, Janet, Pradines, Wallon ou Malrieu, s'est considérablement penchée sur l'étude du phénomène émotionnel en abordant l'un ou l'autre de ses aspects (physiologique, comportemental, affectif ou social).

II nous a semblé opportun de resituer ces auteurs et leur contribution particulière à la théorie des émotions. Des remarques émises ci-dessous, il ressort que le caractère adaptatif de l'émotion, reconnu maintenant unanimement, faisait l'objet de débats passionnés au début du XXème siècle. Les controverses étaient liées à des ambiguités profondes, l'une terminologique, relevant des définitions respectives de l'émotion et du sentiment, l'autre portant sur l'origine et la nature de l'émotion et, partant, sur l'affrontement entre thèses intellectualiste et physiologique.

\section{L'émotion: l'équivoque d'une définition et sa conséquence}

Le concept d'émotion a souffert au début du siècle dernier d'un manque de clarté dans l'emploi respectif des termes émotion, sentiment, passion et états affectifs, ceux-ci étant assimilés l'un à l'autre ou employés l'un pour l'autre en fonction de leur utilisateur. En insistant sur le rôle primordial du sentiment dans l'adaptation, l'émotion était dotée d'un revers négatif: elle 
est une désorganisation et une désadaptation. En vertu de sa particularité quantitative, son intensité, elle a été perçue comme une forme explosive de l'affectivité qui, en envahissant le champ de la conscience, provoque un retour aux automatismes préformés. Elle est une intensité «en plus», «en trop» dans le comportement humain ou encore le «raté d'une régulation sentimentale» (Pradines, 1954).

Si la proposition suivante: «Réaction organisée et utile à une situation donnée» apparaît définitionnelle de l'émotion pour la plupart des théoriciens contemporains, elle correspondait chez Janet à la notion de sentiment. En effet, Janet (1926), puis également Pradines (1954) octroient au sentiment, non à l'émotion, une fonction régulatrice, un caractère fonctionnel et adaptatif. Selon ces auteurs, le sentiment est ce qui nous donne à l'égard des objets une règle d'action parfaitement adéquate; il a pour rôle de mettre notre psychisme en harmonie avec les circonstances, de l'orienter en fonction de ce qu'elles nous offrent et de nous inspirer des conduites efficaces. Wallon, Malrieu ou encore Bergson vont plus loin, en associant le phénomène émotionnel au développement psychique et à la régulation des comportements sociaux.

\section{Une nécessité adaptative: l'émotion et sa nature sociale}

Les doctrines de Wallon (1934, 1938) et de Malrieu (1952) ont évoqué principalement le contexte social et interactionnel associé au phénomène émotionnel. Pour Wallon, dès les premiers jours de vie de l'enfant, les réactions motrices et les attitudes posturales font naître des émotions auxquelles le milieu est appelé à répondre. Ce sont d'elles que procèdent les premières régulations du comportement, puis les progrès des habitudes motrices. La valeur fonctionnelle des émotions chez Wallon tient à ce qu'elles permettent de superposer aux automatismes la diversité des réactions affectives comme moyens d'expression. Selon Malrieu également, leur fonctionnalité s'exprime au travers de leur action sur autrui.

Ainsi, bien loin d'être de simples réactions désorganisatrices, les émotions sont au contraire décisives pour l'adaptation de l'individu et ceci, dès sa naissance. Elles continuent à l'être, par la suite, en tant qu'adaptations circonstanciées à des modèles sociaux. Pour Wallon ou Malrieu, la société exerce une influence sur la différenciation et la reconnaissance des émotions, 
sur leur expression et leur gestion interindividuelle. D'une part, l'acteur social doit accorder ses expressions émotionnelles aux impressions qu'il souhaite produire, en vertu de «codes sociaux», l'idéal étant qu'il y ait harmonie entre les éprouvés, leur expression et les impressions produites. D'autre part, il y a l'ordre social qui impose une dialectique de l'expression émotionnelle et de son usage, tant public que privé. C'est ce dernier point que plusieurs ouvrages classiques français du début du siècle dernier ont traité pour retracer le rapport entre le social et l'émotion, mais leurs discussions des codes émotionnels a principalement concerné l'expression des émotions.

Pour Dumas (1948a), les mots « expression émotionnelle » sont psychologiquement vides de sens et ne peuvent se comprendre que si l'on suppose une vie sociale où l'expression sera interprétée. Par exemple, le sourire figure un geste dont nous pouvons nous servir quand nous le croyons utile, en imitant par des mouvements volontaires un mouvement réflexe. Les expressions émotionnelles, et les émotions, ne sont pas uniquement ce qu'elles sont pour nous, mais aussi ce qu'elles sont pour autrui et leur signification dépend de l'accueil qu'elles reçoivent.

Tout un chapitre de l'Encyclopédie La Pléiade a été également consacré à la nature sociale de l'émotion, retenant que la société fonde son système d'attitudes affectives et détermine ainsi la valeur qu'une communauté attribue aux différents états affectifs. Par exemple, le rire y est défini comme un fait humain institutionnalisé, c'est-à-dire obéissant à des prescriptions sociales, à obligations et des interdits, de même qu'à des exigences relationnelles (voir Clapier-Valadon, 1991). Henri Bergson (1940) avait déjà insisté sur ces particularités du rire, dans son ouvrage intitulé «Le Rire». Ce philosophe indique que, pour comprendre le rire, il faut le replacer dans son milieu naturel de production qui est la société; le rire est un geste social: il est humour, moquerie, ironie.... et poursuit un but utile, celui de «perfectionnement de la société», parce qu'il fixe une réglementation humaine, en informant les individus de ce qu'il faut faire en cas d'émotion.

\section{Origine et nature de l'émotion: l'âme et le corps \& l'opposition entre thèses intellectualistes et physiologiques}

Dès l'aube des premiers propos sur les émotions, deux thèses sont nées et se sont affrontées, l'une partisane d'une origine purement mentale des 
émotions et l'autre, adepte d'une origine organique. Pour la première, les émotions sont dérivées et fonction de la connaissance, pour la seconde, elles ont leur racine dans les besoins et les instincts. Le problème devient que, ce faisant, il y a toujours réduction des émotions en états organiques ou intellectuels, impliquant toujours le sacrifice d'un des facteurs et l'impossibilité d'expliquer leurs rapports. Cet état de fait brouille la discussion du caractère adaptatif vs. non adaptatif de l'émotion.

Dans la théorie de Dumas (1900, 1948ab), cependant, apparaissent un dépassement de cette dichotomie et une prise en compte du rapport âme-corps en tant qu'unité vécue, ce qui justifie, en partie au moins, la plaidoirie de celui-ci en faveur du caractère adaptatif de l'émotion. Vu l'état des connaissances en son temps, Dumas a fourni un travail impressionnant pour caractériser les états organiques, dans leurs aspects physiologiques, psycho-chimiques et psycho-mécaniques, durant l'émotion. Cet auteur reconnaît que les faits physiologiques n'ont pas de valeur explicative suffisante des phénomènes émotionnels et que c'est une erreur de méconnaitre la signification et l'efficacité des facteurs proprement psychiques. A cheval entre thèse physiologique et thèse intellectualiste, Dumas définit l'émotion comme se scindant en deux temps: un moment de surprise et un moment d'émotion spéciale. La surprise, c'est le choc produit par ce qui est nouveau et inattendu auquel répondent des modifications organiques, physiologiques, et, au niveau des manifestations motrices, des mouvements organisés remplaçant réflexes et mouvements automatiques. Parallèlement à ce désordre physique s'instaure un désordre mental: il y a convergence de la pensée vers la cause de ce trouble. Une représentation va intervenir entre le fait nouveau et les habitudes particulières que ce fait nouveau désorganise. Aux représentations négatives, relatives à la désorganisation, se mêlent des représentations positives, à la recherche d'orientations nouvelles des habitudes physiques et des habitudes mentales gênées. Dans cette perspective, l'émotion autorise des variations adaptatives ou des adaptations nouvelles, puisqu'elles modifient les instincts et les habitudes, physiques et mentales.

Dans cet article, nous défendrons l'idée que l'émotion peut être considérée comme un processus de composants qui englobe tous les sous-systèmes organiques et qui est principalement dirigé par des changements dans l'évaluation de l'information reçue. Ce point de vue est incompatible avec une conception de l'existence de systèmes cognitif et affectif séparés, une idée que nous appellerons l'erreur de Platon. II est également 
incompatible avec l'opinion selon laquelle il est un nombre limité d'émotions de base ou fondamentales, ayant chacune un pattern spécifique de réponses physiologiques et expressives. Ces deux incompatibilités seront exprimées sous forme de propositions et étayées par des arguments conceptuels ainsi que des données empiriques.

\section{Les émotions sont différenciées par un traitement évaluatif de l'information dans le système mental. Il est peu probable qu'il y ait un système de laffect séparé}

Dans son modèle tripartite de l'âme, Platon a postulé l'existence de modèles structurels parallèles pour l'esprit humain et la société, en mettant l'accent sur:

(I) une rigide séparation entre les classes sociales et, en conséquence, les composants de l'âme,

(2) la supposition d'un antagonisme entre ces composants, et

(3) la suprématie éthique de la cognition et donc de la classe des philosophes/rois.

Ce modèle propose que le comportement est fortement affecté par trois déterminants: un élément appétitif ou impulsif, un élément de pensée et de raison et, entre les deux, un élément capable de réfréner les impulsions et désirs et capable également de recevoir des ordres de la pensée et de la raison. Cet élément intermédiaire était appelé «thumos», interprétable comme un facteur de l'émotion spirituelle la plus idéale se manifestant en tant que ressentiment, et contre les transgressions d'autrui, et contre celles de nos propres appétits. II convient de noter que ce composant du modèle de Platon, plus tard généralisé à l'émotion ou l'affect en général, était à l'origine réduit à une fonction de «chien de garde» au service de la sagesse et du jugement rationnel. Le bien-être personnel, selon Platon, suppose un fonctionnement harmonieux, rationnellement coordonné de ces éléments (impulsif, cognitif et «thumos»).

Ce modèle n'était pas universellement accepté et a attiré de virulentes critiques, même de la part des élèves de Platon, tel Aristote. Néanmoins, il a influencé virtuellement tout penseur qui s'est depuis lors intéressé à l'esprit humain. II exerce encore quelque influence sur la théorie et la recherche en psychologie actuelle, comme cela peut être mis en évidence par les controverses récentes sur les relations entre cognition et émotion. 
Pour notre part, nous soutiendrons que la distinction platonicienne entre cognition, émotion et conation est dépassée dans son utilité et peut gêner sérieusement, en plus de provoquer de futiles débats, les progrès vers des approches intégratives dont il se fait pour l'heure un besoin urgent pour l'étude de l'activité mentale.

Dans le contexte d'un modèle de processus composants, Scherer (1984a,b,c, 1986, 1993b, 2000, 200 I) a suggéré de définir l'émotion comme un épisode de synchronisation temporaire des principaux sous-systèmes du fonctionnement organique représenté par cinq composants (cognition, régulation physiologique, motivation, expression motrice et sentiment) en réponse à l'évaluation d'un stimulus, externe ou interne, comme pertinent aux intérêts centraux de l'organisme. Bien que les différents sous-sytèmes ou composants opèrent relativement indépendamment les uns des autres durant des épisodes non-émotionnels, en ce qui concerne leur fonction respective dans la régulation globale du comportement, ils travaillent à l'unisson dans des situations d'urgence, les épisodes émotionnels.

L'épisode émotionnel est ainsi conçu comme débutant avec la mise en route de la synchronisation s'ensuivant d'une évaluation du stimulus particulier et cessant avec le retour à un fonctionnement indépendant de ces sous-systèmes (bien que ces systèmes diffèrent dans leur rapidité de réponse et de traitement). Puisque l'on s'attend à ce que l'évaluation du stimulus affecte chaque sous-système directement et compte tenu que tous ces sous-systèmes sont définis comme étant hautement interreliés durant l'épisode émotionnel, la régulation est complexe et englobe de nombreux processus rétro- et pro-actifs.

Pour plusieurs raisons, le sentiment subjectif pourrait figurer le composant le plus important de l'émotion. Nous pourrions en effet nous demander si nous pouvons prétendre avoir une émotion si nous n'avons pas une expérience consciente de celle-ci, un sentiment spécifique que nous pouvons ensuite étiqueter avec un concept verbal approprié. II semble raisonné de considérer le composant sentiment comme une réflexion des changements se produisant dans tous les sous-systèmes (voir Scherer, 1993a, 200 I). Ces sous-systèmes gagnent en effet leur signification principalement à travers l'interprétation des processus d'évaluation cognitive.

Cependant, dans la supposition d'un traitement «cognitif», il reste hautement probable que tous les processus d'évaluation ne soient pas accessibles à la conscience. En effet, certaines des réflexions des systèmes 
neurophysiologique et moteur, aussi bien que les changements de motivation, peuvent ne pas être complètement accessibles à la mémoire. Il est donc nécessaire de distinguer grossièrement dans le sentiment une part inconsciente et une part consciente constituant la représentation globale des facteurs intervenant dans un épisode émotionnel (c'est-à-dire le traitement cognitif des antécédents et les réactions suscitées dans les différentes modalités). On peut par-là même supposer que la part consciente des sentiments va au-delà du matériel inconscient rendu conscient. En raison d'un traitement cognitif actif et constructif, elle pourrait être partiellement construite sur la base de schémas, scripts et représentations sociales. Donc, comme montré dans la Figure I (adapté de Kaiser \& Scherer, 1998), nous pouvons représenter ces parties du composant sentiment par deux cercles de Venn se recoupant partiellement.

Un troisième cercle, chevauchant en partie les deux autres, semble indiqué pour représenter la verbalisation de la partie consciente du sentiment. A nouveau, l'usage d'étiquettes linguistiques ou d'expressions pour décrire la part consciente du sentiment ne couvrira pas toute la zone consciente, notamment du fait de l'absence de labels verbaux appropriés. Un tel usage ajoutera un surplus de sens, c'est-à-dire un ajout de contenu, vue la signification dénotationnelle et connotationnelle des concepts utilisés dans la verbalisation. Ces aspects de signification qui seraient fonction des règles du langage pourraient ne pas toujours être complètement appropriés à l'état de sentiment conscient.

L'approche par les cercles de Venn conduit donc à 7 aires ou aspects du sentiment:

(I) un chevauchement total de l'évaluation inconsciente et consciente verbalisées de manière appropriée;

(2) une représentation inconsciente est consciemment représentée mais ne peut être verbalisée;

(3) une évaluation inconsciente est intuitivement verbalisée sans être consciemment verbalisée;

(4) une représentation consciente construite, basée sur aucune évaluation inconsciente, est verbalisée - le cas typique du stéréotype;

(5) une évaluation inconsciente reste inaccessible;

(6) une évaluation consciente construite n'est pas verbalisée;

(7) un surplus de sens est fourni par l'usage d'étiquettes verbales, non basé sur une représentation consciente. 

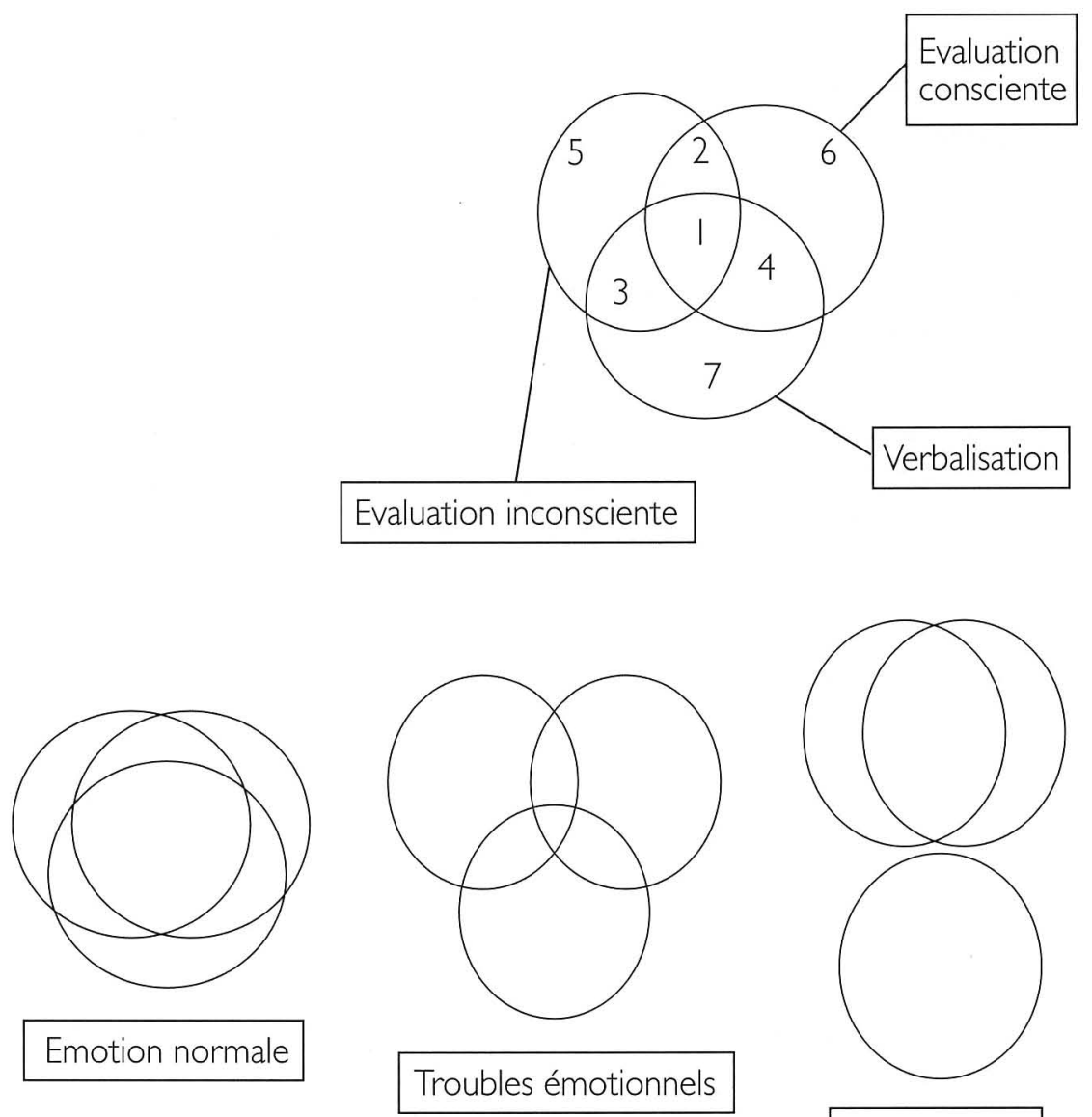

Alexithymie

Figure I - Aires du sentiment, illustrées par les cercles de Venn

Sous des conditions normales, les trois aires du sentiment, illustrées pour des émotions normales dans la Figure I par les cercles de Venn, tendent à se recouper plutôt fortement. En d'autres termes, une grande part du traitement émotionnel est accessible à la conscience, en particulier l'évaluation cognitive d'événements pertinents, donnant une signification appropriée à l'expérience affective, et une grande part peut être adéquatement verbalisée aux fins de communication sociale. 
Les désordres affectifs pourraient être caractérisés par une dissociation augmentée de ces aspects du sentiment. En effet, les processus d'évaluation qui ont généré un état émotionnel particulier pourraient rester inaccessibles à la conscience, du fait de répression ou autres mécanismes de défenses, de déficits attentionnels, de malfonctionnements neurologiques ou d'autres facteurs. Le surplus de signification de la représentation consciente du sentiment pourrait donc être également déterminé dans une large mesure par des facteurs non liés aux processus d'évaluation réels, comme dans le cas d'idées compulsives apparaissant sous formes de schémas ou scripts que la personne tiendrait concernant le soi ou l'environnement social et/ou physique. De même, la verbalisation pourrait représenter seulement une très petite partie du composant sentiment conscient et donc être considérée comme bizarre et inappropriée par l'environnement social, comme par exemple dans le cas d'alexithymie, cité pour exemple de désordre émotionnel (voir Figure I, ainsi que Kaiser \& Scherer, 1998).

\section{Approche fonctionnelle des processus émotionnels}

Le modèle proposé adopte une approche fonctionnelle, en tentant de prendre son point de départ dans les systèmes mental, physiologique et comportemental dont on peut montrer leur asservissement à l'adaptation comportementale - ce qui s'intègre fort dans l'esprit de Darwin. Dans ce sens, la cognition - ou plus généralement le codage de l'information reçue (voir LeDoux, 1989) - joue un rôle déterminant dans la mise en accord des autres sous-systèmes organiques. Ceci n'est pas surprenant puisqu'une adaptation adéquate demande, à tout le moins, un diagnostic approprié des circonstances auxquelles il convient de s'adapter.

L'émotion peut être considérée comme l'une des réalisations principales de l'évolution dans le sens qu'elle remplace la chaîne instinctive Stimulus-Réponse (qui demande seulement un traitement rudimentaire des stimuli déclenchés de façon innée (Lorenz, 1965)) par un mécanisme hautement flexible qui «découple» le stimulus et la réponse. Le processus émotionnel fournit ainsi un temps de latence qui permet un traitement de l'information toujours plus sophistiqué et un choix toujours plus vaste d'alternatives comportementales, mais en préparant néanmoins l'organisme à des réponses d'urgence grossièrement appropriées. 
Le rôle du traitement évaluatif de l'information pour rendre compte de la nature adaptative de l'émotion a été implicitement accrédité par les plus éminents penseurs de l'émotion, tels Aristote, Descartes, Hume et Spinoza. Cependant, la notion d'évaluation (《 appraisal ») comme étant à la racine du déclenchement et de la différenciation de l'émotion est beaucoup plus récente. Dans les années 60, Arnold (1960) et Lazarus (1968) ont explicitement formulé des théories incorporant des critères d'évaluations rudimentaires pour expliquer les conséquences émotionnelles de la confrontation à un événement particulier. Au début des années 80 , plusieurs psychologues ont proposé, indépendamment les uns des autres, des ensembles plus détaillés de dimensions d'évaluation pour interpréter le déclenchement et la différenciation de l'émotion (de Rivera, 1977; Frijda, 1986; Johnson-Laird, \& Oatley, 1989; Mees, 1985; Ortony, Clore, \& Collins, 1988; Roseman, 1984, 1991; Smith \& Ellsworth, 1985, 1987; Scherer, 1981, 1982, 1984a, 1984b, 1986; Solomon, 1976; Weiner, 1986).

Dans les revues comparatives de ces théories de l'évaluation et de la différenciation des émotions, Scherer (1988, 1999b) a tenté de montrer l'important degré de convergence des différentes suggestions théoriques, en particulier concernant les critères centraux postulés dans les différentes approches. Cette ressemblance apparaît comme des plus surprenantes puisque les théoriciens viennent de traditions de psychologie et/ou de philosophie largement différentes. Les empreintes laissées par les théories de l'évaluation et de la différenciation de l'émotion ont généré un corps de recherche qui s'est accumulé depuis lors, les confirmant également par des études empiriques comparatives (Lazarus, \& Smith, 1988; Manstead, \& Tetlock, 1989; Reisenzein, \& Hoffman, 1990; Roseman, Spindel, \& Jose, 1990; Scherer, 1988). L'état actuel de cette approche théorique et de la recherche empirique pertinente est présenté de manière exhaustive dans Scherer, Schorr, \& Johnstone (200I).

20 Nous illustrerons brièvement les principaux tenants des théories de l'évaluation et leurs prédictions, puisque cette approche est de plus en plus acceptée comme un modèle valide d'explication de la plupart des états émotionnels humains. Ce faisant, nous nous centrerons sur le modèle du Groupe de Recherche en Emotions de Genève, la théorie des processus composants de Scherer. Le Tableau I ci-dessous mentionne les critères d'évaluation, dénommés séquences de traitement de la stimulation («stimulus evaluation checks») dans le modèle développé par Scherer (et comparés à quelques autres auteurs). 
TABLEAU I - Critères d'évaluation des séquences de traitement de la stimulation dans le modèle de Scherer et comparaison de ceux-ci avec les critères d'autres théoriciens de l'appraisal

\begin{tabular}{|c|c|}
\hline $\begin{array}{l}\text { Séquence de traitement } \\
\text { de la stimulation (Scherer) }\end{array}$ & $\begin{array}{c}\text { Appraisal dimensions (Frijda, } \\
\text { Ortony/Clore, Roseman, Smith/Ellsworth, } \\
\text { Solomon, Weiner) }\end{array}$ \\
\hline Nouveaute & Change, attentional activity(?) \\
\hline Soudaineté & 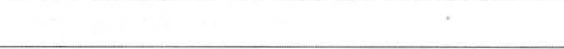 \\
\hline Familiarité & Familiarity \\
\hline Prévisibilité & Unexpectedness \\
\hline Agrement intrinseque & Valence, appealingness, pleasantness \\
\hline \multicolumn{2}{|l|}{ Rapports aux buts } \\
\hline Pertinence & $\begin{array}{l}\text { Focality, appetitive/aversive motivation, } \\
\text { scope/focus, importance }\end{array}$ \\
\hline $\begin{array}{l}\text { Degré de certitude dans la prédiction } \\
\text { des conséquences }\end{array}$ & $\begin{array}{l}\text { Certainty, probabillity, likelihood, } \\
\text { predictability }\end{array}$ \\
\hline Attente & Presence, prospect realization \\
\hline Opportunité & $\begin{array}{l}\text { Open/closed, desirability, motive } \\
\text { consistency, goal/path obstacle, evaluation }\end{array}$ \\
\hline Urgence & Urgency, proximity \\
\hline \multicolumn{2}{|l|}{ Pontentiel de maitrise } \\
\hline Causalité: interne & $\begin{array}{l}\text { Intent/self-other, agency, responsability, } \\
\text { locus of causality }\end{array}$ \\
\hline Causalité: externe & Agency, stability \\
\hline Contrôle & Modifiability, controllability \\
\hline Puissance & Controllability, power \\
\hline \multicolumn{2}{|l|}{ Ajustement } \\
\hline \multicolumn{2}{|l|}{ Accord avec les standards } \\
\hline Standards externes & Value relevance, legitimacy, fairness \\
\hline Standards internes & Blameworthiness \\
\hline
\end{tabular}

Quant au Tableau 2, elle présente un exemple des prédictions qui peuvent être générées sur la base d'une théorie de l'évaluation de ce type. L'idée générale est que l'évaluation subjective d'une situation, d'un événement ou d'un stimulus, sur la base de ces critères d'évaluation, détermine la nature 
(c'est-à-dire à la fois la qualité et l'intensité) de la réaction émotionnelle (Tableau 2, exemples pour colère, peur et tristesse). II convient ici de préciser que ces critères n'ont pas à correspondre à la nature objective de la situation - en fait, l'écart entre évaluation subjective et nature objective de la situation figure souvent à la base de désordres affectifs, voir Scherer, 1987).

TABLEAU 2 - Exemples de prédictions générées sur la base du modèle des processus composants

\begin{tabular}{|c|c|c|c|}
\hline $\begin{array}{c}\text { Séquence de traitement } \\
\text { de la stimulation }\end{array}$ & Colère/Rage & Peur/Panique & Tristesse \\
\hline \multicolumn{4}{|l|}{ Nouveaute } \\
\hline Soudaineté & élevée & élevée & basse \\
\hline Familiarité & basse & ouvert & basse \\
\hline Prévisibilité & basse & basse & ouvert \\
\hline Agrement intrinseque & ouvert & & \\
\hline \multicolumn{4}{|l|}{ Rapports aux buts } \\
\hline Pertinence & ordre & corps & ouvert \\
\hline $\begin{array}{l}\text { Degré de certitude dans la prédiction } \\
\text { des conséquences }\end{array}$ & très élevé & élevé & très élevé \\
\hline Attente & dissonante & dissonante & ouvert \\
\hline Opportunité & enrayée & enrayée & enrayée \\
\hline Urgence & élevée & très élevée & basse \\
\hline \multicolumn{4}{|l|}{ Potentiel de maitrise } \\
\hline Causalité: interne & autrui & autrui/nature & ouvert \\
\hline Causalité: externe & intentionnelle & ouvert & $\begin{array}{l}\text { Chance } \\
\text { /négative }\end{array}$ \\
\hline Contrôle & élevé & ouvert & très bas \\
\hline Puissance & élevé & très basse & très basse \\
\hline Ajustement & élevé & bas & moyen \\
\hline \multicolumn{4}{|l|}{ Accord avec les standards } \\
\hline Standards externes & bas & ouvert & ouvert \\
\hline Standards internes & bas & ouvert & ouvert \\
\hline
\end{tabular}


La plupart des théories de l'appraisal n'ont pas tenté de prédire les processus micro-génétiques impliqués dans l'évaluation des antécédents de l'émotion. Une exception cependant pour ce modèle des processus composants qui postule que les critères d'évaluation proposés (stimulus evaluation checks) se produisent selon une séquence invariante (dans l'ordre indiqué dans les Tableaux I et 2). Cette notion de séquence, basée sur des considérations phylogénétiques, ontogénétiques et micro-génétiques (logiques), soutient l'idée que le processus d'évaluation est constamment opérant avec des évaluations continuellement réalisées pour mettre à jour l'information de l'organisme en fonction d'un événement ou une situation (incluant le besoin et la possibilité d'agir sur ceux-ci). Leur succession très rapide et leur opération continue peuvent expliquer les changements soudains qui se produisent durant les épisodes émotionnels et qui sont souvent basés sur des réévaluations de l'événement ou de nos propres capacités de faire face (cf. «secondary apparaisal» (Lazarus, 1968); voir Scherer 1984a, 1999a, 200 I, pour davantage de détails sur le traitement séquenciel dont il est fait l'hypothèse).

Les théories de l'appraisal sont souvent accusées d'un cognitivisme excessif, l'argument étant que la plupart des émotions semblent se développer beaucoup plus vite et beaucoup plus spontanément qu'elles ne le feraient si on supposait qu'elles étaient des traitements cognitifs approfondis. L'erreur de cette critique réside dans le fait que le traitement évaluatif de l'information est assimilé à un traitement cortical. Cette supposition est, bien sûr, injustifiable. Leventhal et Scherer (1987) ont montré qu'on peut envisager l'existence d'un traitement à plusieurs niveaux avec un organisme à même de s'adapter automatiquement au niveau qui est suffisant et efficace pour les besoins déterminants du moment. Le Tableau 3 montre comment ce traitement à plusieurs niveaux a été conçu par ces auteurs. Aujourd'hui, l'idée d'un traitement à niveaux multiples est fortement. répandu dans les domaines de la recherche sur les émotions, sur le traitement de l'information, et les troubles affectifs (voir Van Reekum \& Scherer, 1997, pour une synthèse).

Il est difficile d'accepter des fonctions ou mécanismes postulés théoriquement qui ne sont pas supportés, ou voire même contredits, par l'organisation d'un substrat.

Les développements théoriques sur la nature du processus d'évaluation et les tentatives éventuelles de modélisation en un paradigme de réseau 
TABLEAU 3 - Niveau de traitement des checks (SECs)

\begin{tabular}{|c|c|c|c|c|c|}
\hline & Nouveaute & $\begin{array}{l}\text { Agrement } \\
\text { intrinseque }\end{array}$ & $\begin{array}{l}\text { Rapports } \\
\text { aux buts }\end{array}$ & $\begin{array}{c}\text { Potentiel } \\
\text { de maitrise }\end{array}$ & $\begin{array}{l}\text { Accord } \\
\text { Avec les } \\
\text { standards }\end{array}$ \\
\hline $\begin{array}{c}\text { Niveau } \\
\text { conceptuel }\end{array}$ & $\begin{array}{c}\text { Attentes: } \\
\text { cause/effet/, } \\
\text { estimation des } \\
\text { probabilités }\end{array}$ & $\begin{array}{c}\text { Evaluation +/- } \\
\text { par anticipation, } \\
\text { souvenir ou } \\
\text { découlement }\end{array}$ & $\begin{array}{l}\text { Buts, plans } \\
\text { conscients }\end{array}$ & $\begin{array}{c}\text { Capacité à } \\
\text { résoudre des } \\
\text { problèmes }\end{array}$ & $\begin{array}{l}\text { Idéal de Soi } \\
\text { évaluation } \\
\text { morale }\end{array}$ \\
\hline $\begin{array}{c}\text { Niveau } \\
\text { schématique }\end{array}$ & $\begin{array}{l}\text { Familiarité: } \\
\text { comparaison } \\
\text { des schémas }\end{array}$ & $\begin{array}{c}\text { Préférences/ } \\
\text { aversions } \\
\text { apprises }\end{array}$ & $\begin{array}{l}\text { Besoins, } \\
\text { mobiles } \\
\text { acquis }\end{array}$ & $\begin{array}{l}\text { Schéma du } \\
\text { corps }\end{array}$ & $\begin{array}{l}\text { Schémas du } \\
\text { Soi et du social }\end{array}$ \\
\hline $\begin{array}{c}\text { Niveau } \\
\text { sensorimoteur }\end{array}$ & $\begin{array}{l}\text { Soudaineté: } \\
\text { stimulatión } \\
\text { intense }\end{array}$ & $\begin{array}{c}\text { Préférences/ } \\
\text { aversions innées }\end{array}$ & $\begin{array}{c}\text { Besoins de } \\
\text { base }\end{array}$ & $\begin{array}{c}\text { Energie } \\
\text { disponible }\end{array}$ & $\begin{array}{c}\text { (Adaptation } \\
\text { empathique ?) }\end{array}$ \\
\hline
\end{tabular}

neuronal trouveront une avancée importante si l'on tient compte des développements récents sur la connaissance des structures anatomiques et des circuits dans le fonctionnement du SNC ayant rapport à l'émotion (Scherer, 1993b). Les travaux de LeDoux (1987, 1989) relatifs aux mécanismes du cerveau impliqués dans l'évaluation de la signification affective du traitement cognitif sont des plus pertinents en la matière. Ses minutieux travaux sur l'identification de circuits fonctionnels dans la perception et l'apprentissage émotionnel chez le rat ont fourni des preuves à l'existence d'une projection directe thalamico-amygdalienne. Les stimuli complexes sont

24 traités dans les noyaux sensoriels thalamiques et reliés aux régions néocorticales en vue d'une analyse et d'une synthèse perceptuelles détaillées. Cependant, des caractéristiques plus simples de ces stimuli activent des cellules thalamiques qui communiquent directement avec l'amygdale et peuvent fournir une évaluation rudimentaire de la signification affective de ces stimuli. Dans ce sens, l'amygdale est conçue comme satisfaisant à une fonction homéostatique générale, en évaluant la signification des informations reçues de sources extéroceptives et intéroceptives et en amorçant rapidement les réponses comportementales et viscérales correspondantes. 
Nous supposons que les deux premiers critères d'évaluation, nouveauté et caractère intrinsèque, du stimulus sont de ces caractéristiques «simples» qui devraient être traitées très rapidement parce que les circuits sensoriels parallèles ont accès à l'amygdale. Elles se produiraient tout à fait au début de la séquence théorique d'évaluation des checks proposée, parce que (a) elles se produisent plus tôt dans la séquence de traitement sensoriel, (b) elles utilisent des circuits plus courts, (c) les cellules thalamiques, dans lesquelles la projection vers l'amygdale se produit, ont des propriétés de règlage relativement faibles (voir LeDoux, 1987, p. 436).

Ces caractéristiques pourraient également expliquer l'argument de Zajonc (1980) pour défendre l'idée d'un système de traitement affectif primaire. La plupart des exemples de Zajonc, si ce n'est tous, impliquent des stimuli qui sont susceptibles d'englober ces aspects de nouveauté et d'agrément intrinsèque. L'évaluation de la signification affective des stimuli sensoriels via les neurones limbiques (par ex. pour la nouveauté ou le caractère agréable) est susceptible de se produire inconsciemment. Si nous en savions davantage sur les structures qui médiatisent le traitement des différentes dimensions d'évaluation, on pourrait alors avoir une meilleure mainmise sur le problème épineux de la conscience dans le traitement émotionnel - particulièrement, en ce qui concerne l'état affectif subjectif.

II convient de relever que la discussion précédente n'implique pas que les structures sub-corticales permettent une évaluation interprétative très approfondie ou sophistiquée. Bien entendu, seules des caractéristiques relativement simples du stimulus peuvent être traitées à ces niveaux. Comme Leventhal et Scherer (1987) ont tenté de le démontrer, la force du système d'évaluation humain réside dans le fait que des niveaux différents d'évaluation sont disponibles et que le traitement peut être adapté à la nature du stimulus ou des besoins interprétatifs de l'organisme. La suggestion d'un processus d'évaluation à plusieurs couches trouve support dans la démonstration de LeDoux (1989) attribuant à l'amygdale et l'hippocampe des fonctions différentiées dans l'évaluation de la signification affective du stimulus. La projection directe thalamico-amygdalienne permet un traitement grossier mais très rapide correspondant au niveau le plus bas, le niveau sensori-moteur du traitement selon le modèle de Leventhal \& Scherer (1987). L'hippocampe est beaucoup plus lent pour répondre aux inputs sensoriels, mais ceux-ci sont intégrés, au travers plusieurs modalités, à un traitement complexe du cortex associatif, avant d'atteindre l'hippocampe, 
ce qui permet, par la suite, un traitement beaucoup plus sophistiqué. Ce circuit pourrait correspondre au niveau schématique du modèle de Leventhal et Scherer (1987), puisque l'on peut supposer que le cortex associatif utilise les patterns en tant que schèmes dans son prétraitement des inputs (voir Figure 2, ci-dessous).

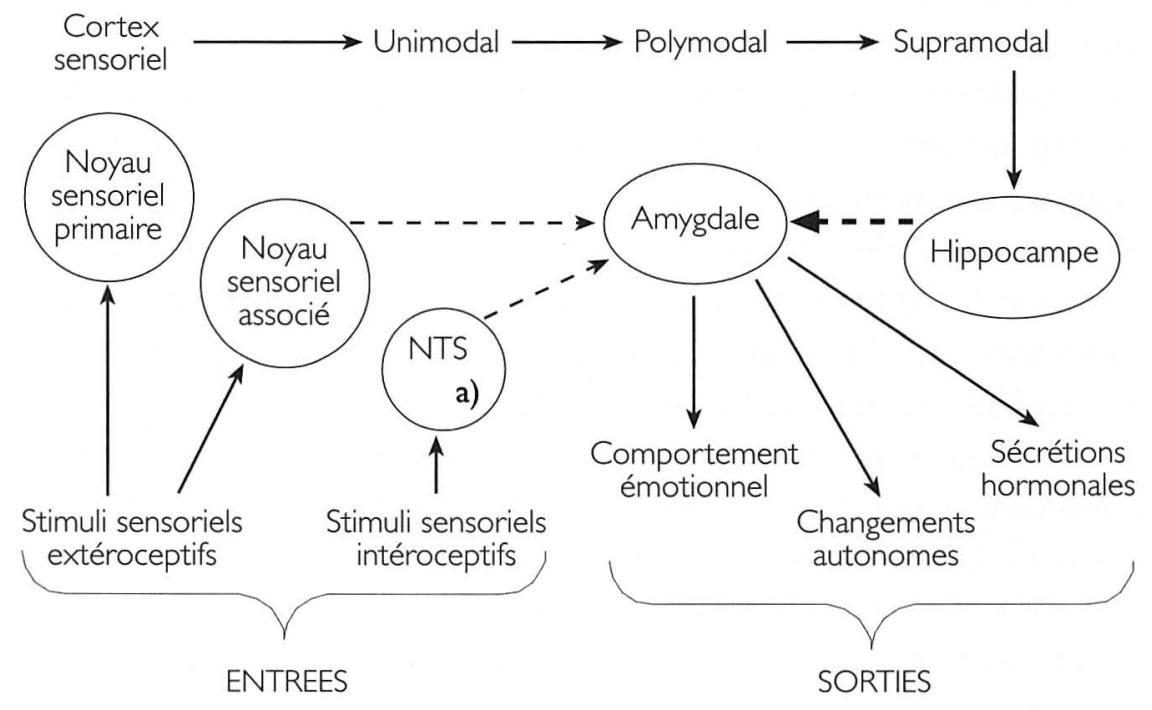

Figure 2 - Niveaux de traitement neuronal de stimuli sonores utilisés dans le conditionnement de la peur chez le rat

(traduit et adapté de LeDoux, 1987)

a) NTS = «nucleus of solitary tract» = noyau de l'appareil cellulaire

Cet argument de «cascades» séquentielles, ou à plusieurs niveaux, de l'évaluation du stimulus est soutenu par de récents travaux menés dans le laboratoire de LeDoux sur les connexions acoustiques de l'amygdale latérale chez le rat. Ces études ont montré que l'information sensorielle, retransmise par les noyaux médiaux et ventraux du corps géniculer médial, atteint directement l'amygdale latérale et à différents intervalles de temps, après un pré-traitement par différentes structures, incluant le cortex périrhinal (LeDoux, communication personnelle). 
Tout cela pour la théorie. Qu'en est-il des faits? II y a actuellement un corps impressionnant de recherches empiriques pour démontrer la validité de ces suggestions hypothétiques (Ellsworth \& Smith, 1988; Frijda, 1987; Frijda, Kuipers, \& ter Schure, 1989; Gehm \& Scherer, 1987; Manstead \& Tetlock, 1989; Reisenzein \& Hoffman, 1990; Roseman, 1984; 1991; Roseman, Spindel, \& José, 1990; Smith \& Ellsworth, 1985, 1987; Tesser, 1990; Weiner, 1986, ainsi que Scherer, 1999b pour un survol). Nous fournirons deux brèves illustrations tirées du programme de recherche de notre laboratoire de Genève.

Dans le cadre d'une étude inter-culturelle à grande échelle (Scherer \& Wallbott, 1994), 2921 répondants de 37 pays ont dû se rappeler d'expériences récentes de joie, tristesse, peur, colère, dégoût, honte et culpabilité et répondre à des questions concernant leur évaluation du comportement ou événement ayant suscité l'émotion. Les résultats obtenus soutiennent la plupart des prédictions du modèle des processus composants, mais pas toutes. Les analyses discriminantes suggèrent qu'un nombre relativement petit de dimensions d'évaluation pourrait être suffisant pour classifier les émotions principales avec un degré de précision appréciable (voir Tableau 4).

TABLEAU 4 - Matrice des résultats résultants des analyses discriminantes

\begin{tabular}{c|c|r|r|r|r|r|r|r}
\hline \multirow{2}{*}{$\begin{array}{c}\text { Emotion } \\
\text { réelle }\end{array}$} & \multirow{2}{*}{$\begin{array}{c}\text { Nbre } \\
\text { de cas }\end{array}$} & \multicolumn{7}{|c}{ Emotion prédite } \\
\cline { 3 - 9 } & Joie & Peur & Colère & Tristesse & Dégoût & Honte & Culpabilité \\
\hline Joie & 530 & 98.3 & .9 & .0 & .2 & .0 & .4 & .2 \\
Peur & 352 & 4.0 & 16.5 & 19.6 & 21.3 & 10.8 & 14.2 & 13.6 \\
Colère & 600 & 1.3 & 8.8 & 47.0 & 17.7 & 12.3 & 7.3 & 5.5 \\
Tristesse & 365 & 3.0 & 8.5 & 19.2 & 36.7 & 9.0 & 13.7 & 9.9 \\
Dégoût & 449 & 2.0 & 13.8 & 33.2 & 12.5 & 23.8 & 7.3 & 7.3 \\
Honte & 546 & 5.1 & 9.0 & 16.1 & 11.0 & 7.0 & 26.4 & 25.5 \\
Culpabilité & 542 & 3.5 & 5.9 & 15.3 & 9.4 & 6.1 & 23.1 & 36.7 \\
\hline
\end{tabular}


Les comparaisons inter-culturelles ont montré que l'effet principal de l'émotion explique davantage de variance que l'effet principal du pays ou l'interaction émotion $X$ pays. Ceci insinue que, bien que le mécanisme d'évaluation et les critères principaux semblent être quasi universels, il y a des différences culturelles dans l'ampleur d'implication des critères d'évaluation spécifiques pour la différenciation des émotions.

L'analyse des facteurs culturels qui peuvent expliquer ces différences démontre l'importance des systèmes de valeurs, tels l'individualisme ou le collectivisme, et des croyances culturelles. Par exemple, les participants Africains ont attribué un degré d'immoralité à événements émotionnels beaucoup plus fort que les participants d'autres parties du monde. II semble que cette tendance soit liée aux croyances au surnaturel dans les pays Africains (Scherer, 1997).

Le Groupe de Recherche en Emotions de Genève a commencé à explorer les techniques développées par la tradition de l'intelligence artificielle pour examiner les manières par lesquelles l'évaluation des antécédents de l'émotion peut aller au-delà du paradigme établi consistant à établir des preuves corrélationnelles entre les rapports verbaux d'expériences émotionnelles verbalement étiquetées et les dimensions d'évaluation inférées. Dans une première étude, Scherer (1993b) a montré qu'une approche «système expert» peut être utilisée pour examiner combien et quels types spécifiques de dimensions d'évaluation sont requises pour diagnostiquer de façon satisfaisante les états émotionnels rapportés par les utilisateurs de ce système. Les données ont montré que leur précision dépasse largement ce qui pourrait être imputé à la chance (voir Figure 3). L'approche «système expert» mène à des suggestions très précises qui ont permis des reformulations et modifications des assomptions théoriques, de même qu'à des nécessités de changements des hypothèses, au vu de preuves

28 empiriques contradictoires. En conséquence, cette approche pourrait fournir un principe de comparaison des théories rivales dans ce domaine et apporter davantage de convergence sur ce sujet.

En collaboration avec Pascal Edwards, Susanne Kaiser et Thomas Wehrle, nous sommes en train d'essayer d'améliorer et d'élargir les algorithmes de l'ordinateur et de développer des outils de simulation pour le développement d'une théorie et l'analyse exploratoire de données. Le prototype d'un tel outil a été élaboré: le GATE - Geneva Appraisal Theory Environment - qui permet, par l'utilisation d'extension graphique, l'évaluation interactive des 


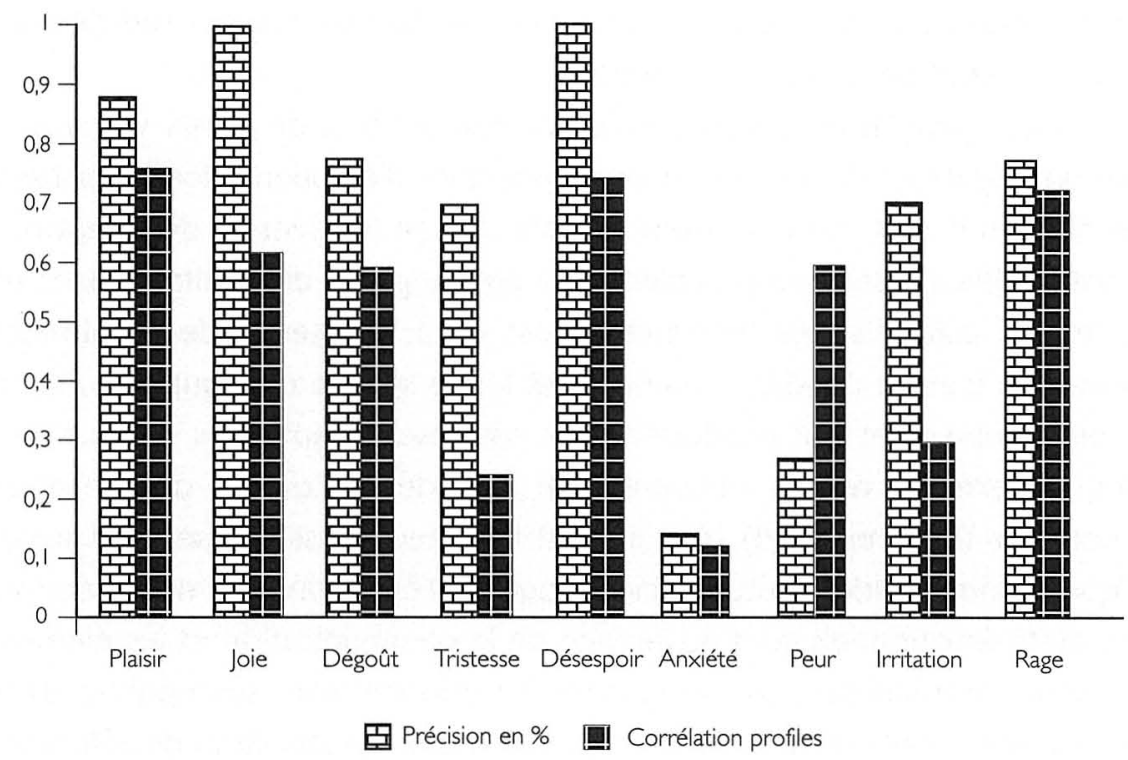

Figure 3 - Précision diagnostique du système expert GENESE, basé sur Scherer, 1993b. (Précision en \% = Pourcentage des prédictions correctes du système expert; Corrélation profiles $=$ Pearson $r$ entre les profils moyens empiriques obtenus et les profils théoriquement prédits sur la base de 15 checks).

effets de changements dans les prédictions théoriques. De façon générale, nous pensons qu'il est utile d'aller au-delà d'une méthodologie classique et d'employer de nouveaux paradigmes de recherches en incluant des simulations par ordinateur et des modèles d'agents autonomes (Wehrle \& Scherer, 2001).

\section{Les émotions constituent des états d'un système organisé de façon} complexe plutôt que des programmes de réponses préfigurées

Une controverse maintenant classique en psychologie des émotions concerne le nombre et le type d'émotions de base caractérisées par des patterns de réponses typiques (voir le chapitre spécial Cognition et Emotion, Vol. 6, 1992). Nous soutiendrons que beaucoup des arguments produits dans 
cette controverse sont égarants et que nous devons changer radicalement notre conception d' «une émotion».

Un des problèmes les plus insidieux est l'emploi de labels verbaux en tant qu'indicateurs du nombre et de l'importance d'émotions «fondamentales». Le fait qu'il soit relativement simple de se référer à des réponses émotionnelles assez comparables, dans des langages différents et dans des contextes culturels très hétéroclites, est dû à l'existence de nombreuses similarités dans la condition humaine et la vie sociale qui sont évaluées de façon identique et qui produisent des réponses adaptatives comparables. Ces patterns universels peuvent être considérés comme des émotions «modales» (Scherer, 1994). Mais il serait trompeur d'user d'une terminologie laïque comme guide à l'étude scientifique de l'émotion. Les mots décrivant des états émotionnels sont au service de la communication et les éléments qui sont communiqués sur un épisode émotionnel sont susceptibles d'être fort variables, car pouvant s'étendre à toute une constellation de références aux antécédents de la situation, allant du type d'évaluation, de l'état physiologique jusqu'aux caractéristiques complexes du sentiment. Malheureusement, la communication scientifique, tout comme notre langage quotidien, dépend pour ses références de moyens verbaux et nous avons dès lors de sérieux problèmes à nous affranchir de ce corset.

II convient entre parenthèses de souligner qu'il serait également trompeur de considérer le fait que les étiquettes verbales et les états affectifs peuvent être appréhendés facilement au travers un petit nombre de dimensions, telles la valence ou l'activité qui ont été amplement employés depuis Wundt (1903; voir Osgood et al., 1975) comme base au développement des théories de l'émotion (par ex. Russell, 1980). Une fois encore, les dimensions du sentiment et sa sémantique sont des images, plutôt que les discriminants de l'émotion. Ils représentent des dispositifs descriptifs qui sont intéressants en eux-même mais qui ne nous fournissent pas d'éclaircissements sur la nature de l'émotion.

Nous croyons également qu'il est déroutant de définir les émotions de base essentiellement à partir de leur configuration des expressions faciales (Ekman, 1984, 1992; Izard, 1971, 1977; Tomkins, 1984). Bien qu'il y ait de bonnes preuves que des acteurs laïques ou professionnels peuvent produire de façon précise des expressions faciales pouvant être reconnues même au travers différentes cultures, ceci n'apporte pas la preuve que l'expression faciale de l'émotion est en effet hautement standardisée et limitée à quelques patterns prototypiques. Quelques travaux récents, en dépit du fait qu'il y ait 
recours à des acteurs qui pourraient être considérés comme s'appuyant sur des expressions hautement schématiques, trouvent un degré assez important de variabilité dans l'expression faciale affichée (Galati, Scherer, \& Ricci-Bitti, 1997).

En suivant ces mises en matière, démarrées par Duchenne et Darwin, puis poursuivies par les contributions de Sancte de Sanctis (1904) et de la psychologie de l'expression allemande (Lersch, 1932/197I; Schänzle, 1939), on peut poser l'argument que l'expression faciale est componentielle, avec des unités musculaires individuelles reflétant soit l'évaluation, soit les tendances à l'action - au service d'une fonction adaptative (Frijda, \& Tcherkassof, 1997; Scherer, 1992; Smith, \& Scott, 1997). La configuration de l'expression faciale dans une telle perspective est le résultat cumulatif de mouvements «en pièces détachées» qui, étant donné la large variabilité des patterns d'évaluation et des combinaisons de tendances à l'action, sont susceptibles d'être relativement variables au travers les individus et les situations.

Des arguments semblables peuvent être avancés pour l'expression vocale de l'émotion. Dans une étude récente, Banse et Scherer (1996) ont montré qu'il y a des profils de caractéristiques acoustiques pour différentes émotions, mais que ceux-ci pouvaient être assez dissemblables en fonction de membres différents des familles d'émotion (telles que une colère chaude vs. froide, anxiété vs. panique). De plus, il ressort passablement de variabilité en fonction des acteurs. Par ailleurs, les différences dans la précision avec laquelle les profils vocaux de l'émotion sont reconnus par les juges semblent liées aux aspects fonctionnels des vocalisations de l'émotion (par ex. le dégoût est plutôt faiblement reconnu et la cause présumée est que nous sommes en fait peu susceptibles de maintenir des settings fonctionnels du dégoût dans nos régions oro-pharyngiales et faciales qui se produisent lors de la rencontre de quelque chose de déplaisant).

Comme pour le visage, on peut défendre l'argument que l'expression vocale reflète l'évaluation et les tendances à l'action, plutôt que des expressions de catégorie d'émotion pure. En partant de données récentes sur les fonctions représentatives et symboliques des cris chez les primates (Marler, 1984), Scherer (1986) a soulevé la question de savoir si la vocalisation affective humaine pourrait être également au service de fonctions cognitives représentatives aussi bien que de fonctions émotionnelles-motivationnelles. Un modèle développé antérieurement sur les fonctions de signal, le modèle «Organon» proposé par Bühler (1933, 1934), qui postule des fonctions de signalement, de symptômes et de 
symboles pour tous les signaux, permet de conceptualiser la nature multi-fonctionnelle de l'expression vocale. De plus, une distinction peut être faite entre des facteurs «push» qui «poussent»l'expression affective, principalement via les effets physiologiques, et les facteurs «pull» qui «tirent» leur expression via les modèles socialement médiatisés. Les facteurs «pull» semblent souvent être impliqués dans les fonctions de signalement. Bien que les facteurs «push», aux bases physiologiques, fournissent souvent des symptômes sur l'état de l'émetteur, on peut avancer qu'ils peuvent également servir à des fonctions cognitives représentatives dans le sens qu'ils reflètent les processus cognitifs d'évaluation des antécédents qui ont produit l'état affectif chez l'émetteur (voir Scherer, 1986, pour les détails).

L'étude psycho-physiologique des réponses émotionnelles, où s'est révélée la difficulté de démontrer empiriquement des configurations stables des paramètres physiologiques qui seraient typiques pour certaines émotions, pourrait être aussi résolue par une approche componentielle. De nouveau, en revenant sur la valeur fonctionnelle de la réponse physiologique (par ex. Lacey et al, 1963), il se justifie d'affirmer que les changements physiologiques pourraient être dirigés de manière componentielle: en fournissant l'adaptation physiologique nécessaire au traitement de l'information ou aux tendances à l'action produits par l'évaluation des antécédents de l'émotion (voir Stemmler, 1984).

L'approche componentielle décrite ci-dessus nécessite un nouveau paradigme pour étudier les dépendances et inter-relations du système, étant donné que la notion classique de «programmes expressifs neuro-moteurs» (Ekman, 1972, 1984; Tomkins, 1963) ne semble pas très adéquate pour rendre compte des mécanismes impliqués. Une approche prometteuse serait d'utiliser des approches dynamiques non linéaires (par exemple la théorie du chaos ou de la catastrophe) pour modéliser les caractéristiques de la réponse émotionnelle. La nature transitoire de l'épisode émotionnel, caractérisé par des changements d'état et la synchronisation de tous les sous-systèmes organismiques, peut être comparée aux modèles physiques des oscillateurs couplés, l'état du système étant temporairement contrôlé par des attracteurs étrangers (Redington, \& Reidbord, 1992). De même, les changements abrupts dans l'état émotionnel et les effets de l'état initial, peuvent être joliment modélisés par la théorie de la catastrophe (Zeeman, 1976). Dans ce sens, Scherer (2000) a suggéré d'adopter une approche dynamique et non linéaire à la modélisation de l'émotion. 
En conclusion, en étudiant l'émotion en tant que mode d'opération de l'organisme, dans le sens d'une synchronisation de plusieurs composantes d'un «système opérant», plutôt qu'en tant que système relativement séparé ou séries de programmes neurophysiologiques rigides, on ouvre la voie à un grand nombre de perspectives prometteuses pour les théories et recherches futures, qui convergeraient avec certains changements dans les paradigmes fondamentaux, observables dans la plupart des disciplines actuelles, en direction de complexité et de non-déterminisme.

\section{REFERENCES}

Arnold, M.B. (1960). Emotion and personality. (Vol. I) Psychological aspects. New York: Columbia University Press.

Banse, R., \& Scherer, K.R. (1996). Vocal profiles of affect: Evidence for 14 emotions. Journal of Personality and Social Psychology, 70(3), 614-636.

Bergson, H. (1940). Le rire. Essai sur la signification du comique. Paris : Presses Universitaires de France: Quadrige.

Bühler, K. (1933). Ausdruckstheorie. Jena: Fischer.

Bühler, K. (1934). Sprachtheorie. Jena: Fischer.

Clapier-Valadon, S. (1991). L'homme et le rire. Encyclopédie La Pléiade, Histoire des Moeurs, Vol. III. Paris : Editions Gallimard.

De Rivera, J. (1977). A structural theory of the emotions. Psychological Issues, 10 (4), 178.

Dumas, G. (1948a). La vie affective. Paris: Presses Universitaires de France.

Dumas, G. (1948b). Le Sourire, Psychologie et Physiologie. Paris : Presses Universitaires de France.

Dumas, G. (1900). La Tristesse et la Joie. Paris : Félix Lacan Editeur.

Ekman, P. (1972). Universals and cultural differences in facial expression of emotion. In J.R. Cole (Ed.), Nebraska Symposium on Motivation (pp. 207- 283). Lincoln: University of Nebraska Press.

Ekman, P. (1984). Expression and the nature of emotion. In K.R. Scherer \& P. Ekman (Eds.), Approaches to emotion (pp. 319-344). Hillsdale, N.J.: Erlbaum.

Ekman, P. (1992). An argument for basic emotions. Cognition and Emotion, 6, 169-200.

Ellsworth, P.C., \& Smith, C.A. (1988). From appraisal to emotion: Diffferences among unpleasant feelings. Motivation and Emotion, 12, 27I-302.

Fraisse P. (1969). Motivation, Emotion et Personnalité. In Traité de Psychologie Expérimentale, Vol IX, Presses Universitaires de France, Paris.

Frijda, N. (1986). The Emotions. Cambridge \& New York : Cambridge University Press.

Frijda, N. (1987). Emotion. Cognitive structure and action readiness. Cognition and Emotion, I, $115-143$.

Frijda, N. H., \& Tcherkassof, A. (1997). Facial expressions as modes of action readiness. In Russell, J. A., \& Fernández-Dols, J. M. (Eds.), The psychology of facial expression (pp. 78- 102). Cambridge: Cambridge University Press.

Frijda, N., Kuipers P., \& Ter Schure E. (1989). Relations Among Emotion, Appraisal, and Emotional Action Readiness. Journal of Personality and Social Psychology, 57(2), 212-228. 
Galati, D., Scherer, K. R., \& Ricci-Bitti, P. E. (1997). Voluntary facial expression of emotion: Comparing congenitally blind with normally sighted encoders. Journal of Personality and Social Psychology, 73(6), | 363-1379.

Gehm, Th., \& Scherer, K. R. (1987). Emotionsantezedente Faktoren als subjektive Dimensionierungskriterien emotionsbeschreibender Adjektive. Sprache und Kognition, 2 , $51-63$.

Izard, C.E. (197I). The face of emotion. New York: Appleton- Century-Crofts.

Izard, C.E. (1977). Human emotions. New York: Plenum.

Janet, P. (1926). La pensée intérieure et ses troubles. Compte rendu intégral du cours professé par P. Janet au Collège de France. Psychologie expérimentale et comparée. Paris : Publications A. Chahine.

Johnson-Laird, P.N. \&, Oatley, K. (1989). The Language of emotions: An analysis of a semantic field. Cognition and Emotion, 3, 81-123.

Kaiser, S., \& Scherer, K. R. (1998). Models of 'normal' emotions applied to vocal and facial expression in clinical disorders. In W. F. Flack, Jr. \& J. D. Laird (Eds.), Emotions in Psychopathology, (pp. 81-98). New York: Oxford University Press.

Lacey, J.I, Kagan, J., Lacey, B.C., \& Moss, H.A. (1963). The visceral level: Situational determinants and behavioral correlates of autonomic response patterns. In P.H. Knapp (Ed.), Expression of the emotions in man. New York: International Universities Press, 161-196.

Lazarus, R.S. (1968). Emotions and adaptation: Conceptual and empirical relations. In W.J. Arnold (Ed.). Nebraska Symposium on Motivation (Vol. 16, pp. 175-270). Lincoln, Ne.: University of Nebraska Press.

Lazarus, R.S., \& Smith, C.A. (1988). Knowledge and appraisal in the cognition-emotion relationship. Cognition and Emotion, 2, 281-300.

LeDoux, J.E. (1987). Emotion. In F. Plum \& V. Mountcastle (Eds.), Handbook of physiology. Nervous system, Vol. 5. Higher Functions (pp. 419-459). Washington, DC: American Physiological Society.

LeDoux, J.E. (1989). Cognitive-emotional interactions in the brain. Cognition and Emotion, 3 , 267-289.

Lersch, P. (1932/197|). Gesicht und Seele: Grundlinien einer mimischen Diagnostik. München: Reinhart. Leventhal, H., \& Scherer, K.R. (1987). The relationship of emotion and cognition: A functional approach to a semantic controversy. Cognition and Emotion, I, pp. 3-28.

Lorenz, K. (1965). Ueber tierisches und menschliches Verhalten. Aus dem Werdegang der Verhaltenslehre. München: Pieper.

Malrieu, Ph. (1952). Les émotions et la personnalité de l'enfant. Etudes de Psychologie et de Philosophie, XII. Paris : Librairie Philosophique J.Vrin.

Manstead, A.S., \& Tetlock, P.E. (1989). Cognitive appraisals and emotional experience: further evidence. Cognition and Emotion, 3, 225-240.

Marler, P. (1984). Animal communication: Affect of Cognition? In K.R. Scherer \& P. Ekman (Eds.), Approaches to emotion (pp. 345-368). Hillsddale, N.J.: Erlbaum.

Mees, U. (1985). Was meinen wir, wenn wir von Gefühlen reden? Zur psychologischen Textur von Emotionswörtern. Sprache und Kognition, 1, 2-20.

Ortony, A., Clore, G., \& Collins, A. (1988). The cognitive structure of emotions. Cambridge : Cambridge University Press.

Osgood, C.E., May, W.H., \& Miron, M.S. (1975). Crosscultural universals of affective meaning, Urbana, III, University of Illinois Press. 
Pradines, M. (1954). Sur les conceptions actuelles de l'émotion. La Psychologie du XXème siècle. Paris : Presses Universitaires de France.

Redington, D. J., \& Reidbord, S. P. (1992). Chaotic Dynamics in Autonomic Nervous System Activity of a Patient During a Psychotherapy Session. Biological Psychiatry, 31, 993- 1007.

Reisenzein, R., \& Hoffmann, T. (1990). An investigation of dimensions of cognitive appraisal in emotion using the repertory grid technique. Motivation and Emotion, 14, I-26.

Roseman, I.J. ( 1984). Cognitive determinants of emotion: A structural theory. In P. Shaver (Ed.) Review of personality and social psychology, (Vol. 5, pp. II-36). Beverly Hills, CA: Sage.

Roseman, I.J. (1991). Appraisal determinants of discrete emotions. Cognition and Emotion, 5, |6|-200.

Roseman, I.J., Spindel, M.S., \& Jose, P. E. (1990). Appraisal of emotion-eliciting events: Testing a theory of discrete emotions. Journal of Personality and Social Psychology, 59, 899-9।5.

Russel, J.A. (1980). A circumplex model of affect. Journal of Personality and Social Psychology, $39,161-178$.

Sanctis, S. de (1904). La Mimica del Pensiero. Milano: Sandron

Schänzle, J. (1939). Der mimische Ausdruck des Denkens. Berlin: Bernard \& Graefe.

Scherer, K.R. (198I). Wider die Vernachlässigung der Emotion in der Psychologie. In W. Michaelis (Ed.) Bericht über den 32. Kongress der Deutschen Gesellschaft für Psychologie in Zürich 1980, (pp. 304-317). Göttingen: Hogrefe.

Scherer, K.R. (1982). Methods of research on vocal communication: Paradigms and parameters. In K.R. Scherer \& P.Ekman (Eds.). Handbook of methods in nonverbal behaviour research (pp.136-198). Cambridge: Cambridge University Press.

Scherer, K.R. (1984a). On the nature and function of emotion: A component process approach. In K.R. Scherer, \& P. Ekman (Eds.). Approaches to emotion (pp. 293-328). Hillsdale, N.J.: Erlbaum.

Scherer, K.R. (1984b). Emotion as a multicomponent process: A model and some cross-cultural data. In P. Shaver, \& L. Wheeler (Eds.). Review of Personality and Social Psychology (Vol. 5, pp. 37-63). Beverly Hills, CA: Sage.

Scherer, K. R. (1984c). Les Emotions : Fonctions et composantes. Cahiers de Psychologie Cognitive, 1, 9-39 (repris dans Rimé, B., \& Scherer, K. R. (Eds) (1989). Les Emotions. Neuchâtel-Paris : Delachaux \& Niestlé.

Scherer, K.R. (1986). Vocal affect expression: A review and a model for future research. Psychological Bulletin, 99, 143-165.

Scherer, K.R. (1987). Vocal assessment of affective disorders. In J.D. Maser, (Eds.) Depression and expressive behavior (pp. 57-82). Hillsdale, NJ: Erlbaum.

Scherer, K.R. (1988). Criteria for emotion-antecedent appraisal: A review. In V. Hamilton, G.H. Bower \& N. H. Frijda (Hrsg.), Cognitive perspectives on emotion and motivation. (pp. 89126). Dordrecht: Kluwer.

Scherer K.R. (1993a). Neuroscience Projections to Current Debates in Emotion Psychology. Cognition and Emotion, 7(I), I-4I.

Scherer K.R. (1993b). Studying the Emotion-Antecedent Appraisal Process: An Expert System Approach. Cognition and Emotion, 7 (3/4), 325-355.

Scherer, K.R., \& Wallbott, H.G. (1994) Evidence for Universality and cultural Variation of Differential Emotion Response Patterning. Journal of Personality and Social Psychology, 66(2), 310-328.

Scherer, K. R. (1997). The role of culture in emotion-antecedent appraisal. Journal of Personality and Social Psychology, 73, 902-922. 
Scherer, K. R. (1999a). On the sequential nature of appraisal processes : Indirect evidence from a recognition task. Cognition and Emotion, I3(6), 763-793.

Scherer, K. R. (1999b). Appraisal theories. In T. Dalgleish, \& M. Power (Eds.), Handbook of Cognition and Emotion (pp. 638-663). Chichester: Wiley.

Scherer. K. R. (2000). Emotions as episodes of subsystem synchronization driven by nonlianear appraisal processes. In M. D. Lewis \& I. Garnic (Eds.), Emotion, development, and selforganization : Dynamic systems approaches to emotional development (pp. 70-99). Cambridge \& New York : Cambridge University Press.

Scherer, K. R. (200I). Appraisal considered as a process of multi-level sequential checking. In K. R. Scherer, A. Schorr., \& T. Johnstone (Eds.), Appraisal processes in emotion : Theory, Methods, Research (pp. 92-120). New York and Oxford : Oxford University Press.

Scherer, K. R., Schorr, A., \& Johnstone, T. (Eds.) (200I). Appraisal processes in emotion : Theory, Methods, Research. New York and Oxford: Oxford University Press.

Smith, C.A., \& Ellsworth, P.C. (1985). Patterns of cognitive appraisal in emotion. Journal of Personality and Social Psychology, 48, 8I3-838.

Smith, C.A., \& Ellsworth, P.C. (1987). Patterns of appraisal and emotion related to taking an exam. Journal of Personality and Social Psychology, 52, 475-488.

Smith, C. A., \& Scott, H. S. (1997). A componential approach to the meaning of facial expressions. In J. A. Russell \& J. M. Fernandez-Dols (Eds.), The psychology of facial expression (pp. 229-254). Cambridge \& New York: Cambridge University Press.

Solomon, R.C. (1976). The passions. The myth and nature of human emotion. Garden City, NY: Doubleday.

Stemmler, G. (1984). Psychophysiologische Emotionsmuster (Psychophysiological patterning of emotion). Frankfurt, Germany: P. Lang

Tesser, A. (1990). Smith and Ellsworth's appraisal model of emotion: A replication, extension, and test. Personality and Social Psychology Bulletin, 16, 210-223.

Tomkins, S.S. (1984). Affect Theory. In K.R. Scherer \& P. Ekman (Eds). Approaches to emotion (pp. 63-196). Hillsdale. NJ: Lawrence Erlbaum Associates Inc.

Van Brakel, J. (1994). Emotions: a cross-cultural perspective on forms of life. Social Perspectives on Emotion, 2, 179-237.

Van Reekum, C. M., \& Scherer, K. R. (1997). Levels of processing for emotion-antecedent appraisal. In G. Matthews (Ed.), Cognitive Science Perspectives on Personality and Emotion (pp. 259-300). Amsterdam : Elsevier Science.

Wallon, H. (1938). La vie mentale. Paris : Editions sociales.

Wallon, H. (1934). Les origines du caractère chez l'enfant. Paris : Boivin \& Cie Eds.

Wehrle T., \& Scherer K.R. (200I). Towards computational modeling of appraisal theories. In K. R. Scherer, A. Schorr, \& T. Johnstone (Eds.). Appraisal processes in emotion :Theory, Methods, Research (pp. 350-365). New York: Oxford University Press.

Weiner, B. (1986). An attributional theory of motivation and emotion. New York: Springer.

Wundt, W. (1903). Grundzüge der physiologischen Psychologie, Band 3. Leipzig, Engelmann.

Zajonc, R. B. (1980). Thinking and feeling: Preferences need no inferences. American Psychologist, $35,151-175$.

Zeeman, E. C. (1976). Catastrophe Theory. Scientific American, 234 (4), 65-83. 
(Página deixada propositadamente em branco) 
Série

Investigaçāo

Coimbra

Imprensa da Universidade

Les Presses de L'Université Laval

Les Editions de L'IORC

2004 